\title{
Syndrome de Gorlin-Goltz : du diagnostic au traitement : à propos d'une observation
}

\section{Gorlin-Goltz syndrome : from diagnosis}

to treatment : a case report

\section{Résumé}

\section{MOTS-CLEFS :}

- Syndromede Gorlin-Goltz, kératokyste odontogénique, nævomatose basocellulaire

\section{KEYWORDS:}

- Gorlin-Goltz syndrome, odontogenic keratocyst, nevoid basal cell carcinoma

$\operatorname{AOS} n^{\circ} 285-2017$
Le syndrome de Gorlin-Goltz ou naevomatose basocellulaire est une affection héréditaire, à transmission autosomique dominante, liée à une mutation sur le gène PTCH suppresseur de tumeur ayant comme localisation chromosomique 9q22.3-q31.

C'est un syndrome rare caractérisé par une série d'anomalies de développement et par une prédisposition ò différents cancers.

Le syndrome de Gorlin-Glotz associe plusieurs manifestations cliniques générales dont de nombreux carcinomes et naevi baso-cellulaires, des hyperkératoses palmoplantaires, des anomalies squelettiques, des calcifications ectopiques intracrâniennes, une dysmorphie faciale avec macrocéphalie.

Le médecin dentiste joue un rôle important dans le diagnostic de ce syndrome à travers les signes maxillo-faciaux spécifiques à son expression notamment : les kératokystes odontogéniques, les inclusions et les ectopies dentaires qui peuvent être inaugurales.
Abstract

Gorlin syndrome, also known as nevoid basal cell carcinoma syndrome (NBCCS), is a hereditary condition transmitted as an autosmal dominant trait, due to a mutation in the tumor suppressor gene PTCH mapped to chromosome 9q 22.3-q31

It is a rare syndrome characterized by a series of developmental abnormalities and predisposition to various cancers.

The Gorlin syndrome - Glotz combines several general clinical manifestations including many basal cell carcinomas and nevi, palmar-plantar hyperkeratosis, skeletal abnormalities, intracranial ectopic calcifications, facial dysmorphism with macrocephaly.

The dentist have an important role in the diagnosis of this syndrome through maxillofacial signs specific to its expression including : keratocysts odontogenic, inclusions and dental ectopias that can be inaugural.

\section{Ossama NABIH, Résident en odontologie chirurgicale, CCTD CHU IBN ROCHD} Casablanca,ossama.nabih@gmail.com

Zineb RACHDY, Résidente en orthopédie dento-faciale, CCTD CHU IBN ROCHD Casablanca.

Olaya MEDAGHRI ALAOUI, Professeur de l'enseignement supérieur, Service d'odontologie chirurgicale, CCTD CHU IBN ROCHD Casablanca.

Ihsane BEN YAHYA, Professeur de l'enseignement supérieur, Chef de service d'odontologie chirurgicale, CCTD CHU IBN ROCHD Casablanca. 


\section{INTRODUCTION}

Nous rapportons, le cas d'une patiente âgée de 22 ans, atteinte de ce syndrome depuis 10 ans, suivie au service de chirurgie maxillo-faciale, adressée au centre de consultations et de traitements dentaires par son médecin traitant pour l'exérèse des lésions kystiques mandibulaires.

\section{OBSERVATION}

L'enquête médicale n'a pas révélé d'antécédents familiaux. L'examen général révèle un retard mental et une cyphoscoliose vertébrale (Fig. 1).

L'examen maxillo-facial met en évidence différentes manifestations notamment :

$\checkmark$ un faciès longiligne avec macrocéphalie ;

I une saillie des bosses frontales, un strabisme, un ptosis bilatéral, une base du nez élargie et un hypertélorisme (Fig. 2); $\checkmark$ de multiples petits naevi disséminés sur le visage (Fig. 3) ;

$\checkmark$ des arcades sourcilières proéminentes.

L'examen endobuccal révèle :

I Une inflammation et des dépôts importants de plaque ;

IDes tuméfactions mandibulaires volumineuses, comblant le vestibule au niveau postérieur et antérieur compliquées d'une fistule (Fig. 4);

\Des ectopies et hétérotopies dentaires;

IUne voûte palatine ogivale (Fig. 5).

La radiographie panoramique a mis en évidence de volumineuses images radioclaires mandibulaires et maxillaires d'allures kystiques uniloculaires à limites nettes refoulant les dents adjacentes (Fig. 6).

La présence de lésions maxillaires hautes en rapport avec le sinus et mandibulaires en rapport avec le nerf alvéolaire inférieur nous a poussé à la prescription d'un Dentascan (Fig. 7, 8).

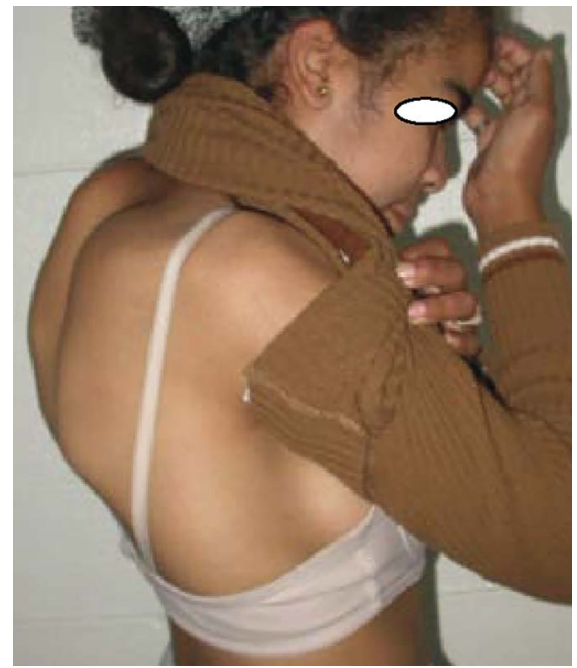

$\triangle$ Fig. 1: Photo de profil objectivant la cyphoscoliose vertébrale.

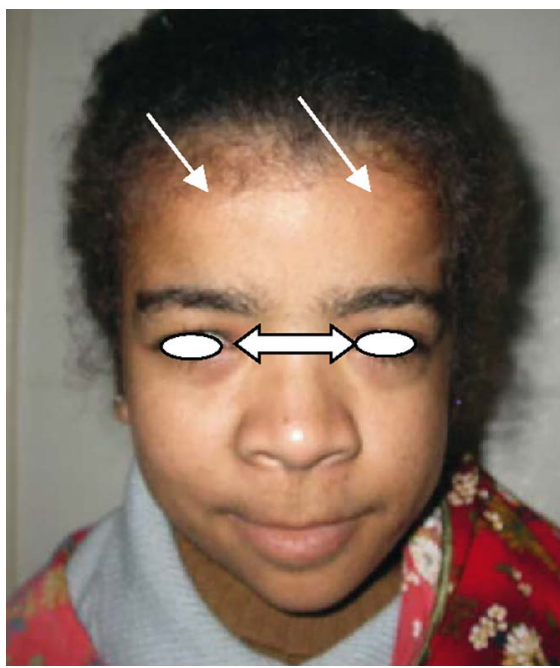

$\triangle$ Fig. 2: Photo de face objectivant la saillie des bosses frontales, le strabisme, le ptosis, la base du nez élargie et l'hypertélorisme.

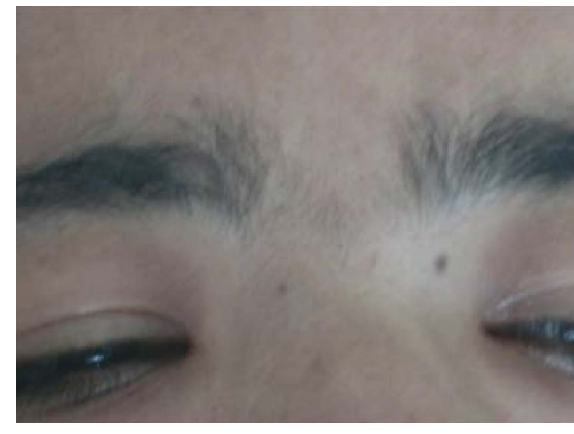

Fig. 3 : Multiples naevi basocellulaires.

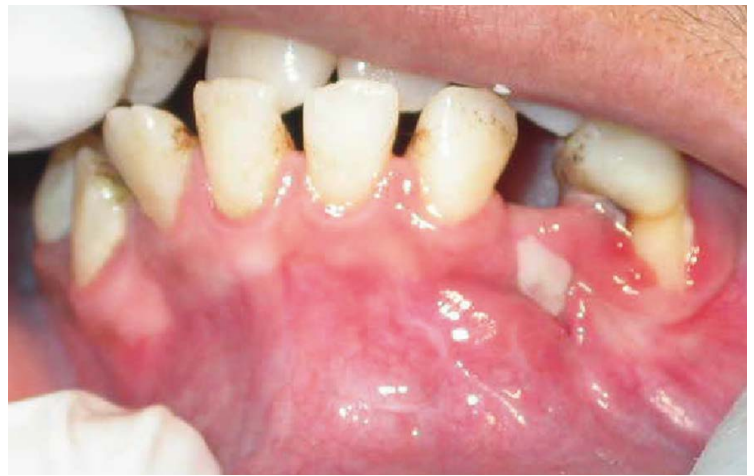

$\triangle$ Fig. 4 : Soufflure de la table externe mandibulaire au niveau antérieur compliquée d'une fistule.

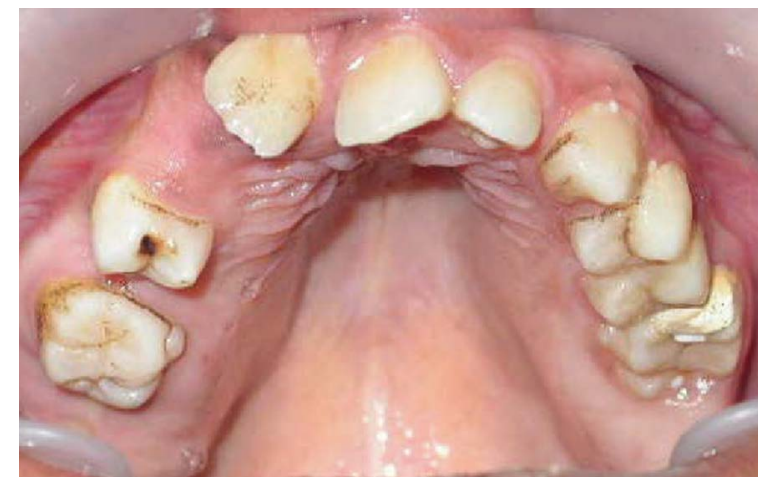

$\triangle$ Fig. 5 : Vue d'avion du maxillaire objectivant la forme ogivale de la voute palatine. 


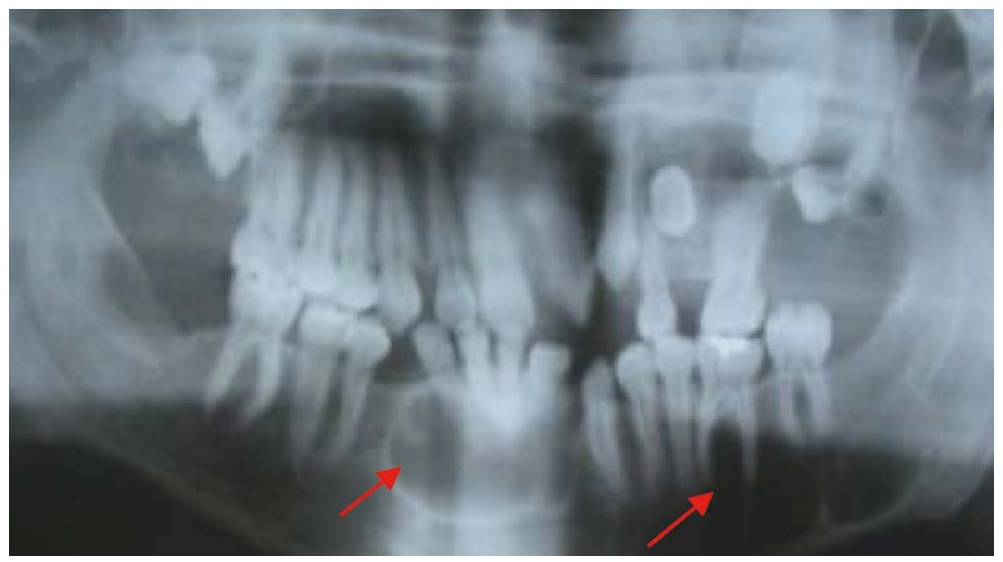

$\triangle$ Fig. 6 : Radiographie panoramique mettant en évidence des images radioclaires d'allure kystique.

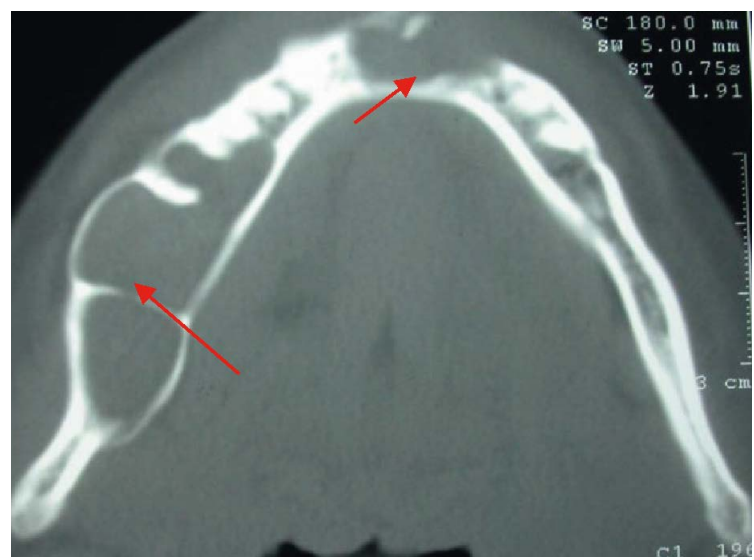

$\triangle$ Fig. 8: TDM : Coupe axiale objectivant les lésions kystiques mandibulaires.

\section{Diagnostic}

L'ensemble des signes cliniques et radiologiques relevés ont permis d'appuyer le diagnostic du syndrome de Gorlin-Goltz.

Le diagnostic présomptif des tuméfactions présentes indiquait des kératokystes

\section{Plan de traitement}

Le plan de traitement de la patiente était comme suit : Motivation à l'hygiène buccodentaire

$\checkmark$ Détartrage et surfaçage

\ Traitement chirurgical des lésions kystiques.

I Prise en charge orthodontique

\section{Prise en charge de la patiente}

La lésion mandibulaire postérieure gauche a été opérée en premier, suivie de l'exérèse du kyste antérieur, sous anésthésie loco-régionale (Fig 9, 10, 11).

L'examen anatomopathologique a confirmé le diagnostic des kératokystes (Fig. 12).

Les kystes maxillaires ont été opérés au service de chirurgie maxillo-faciale.

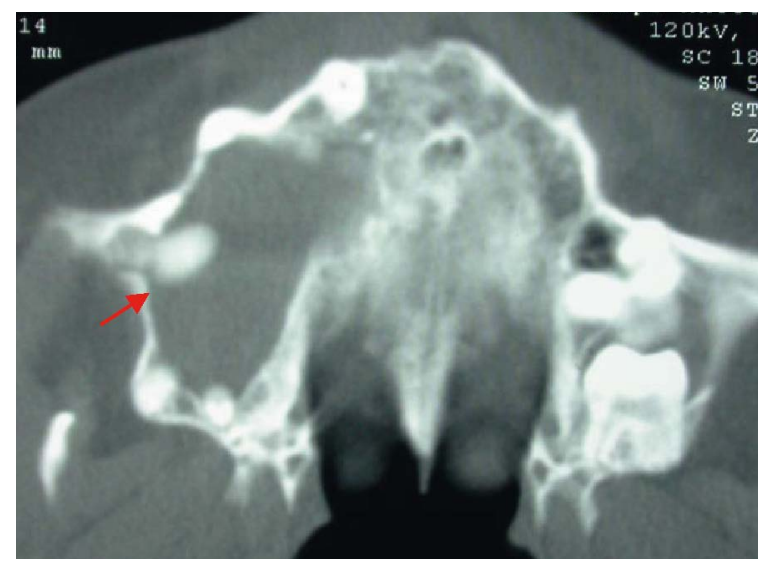

$\triangle$ Fig. 7 : Coupe axiale haute objectivant l'inclusion de la 28 associé à une lésion kystique.

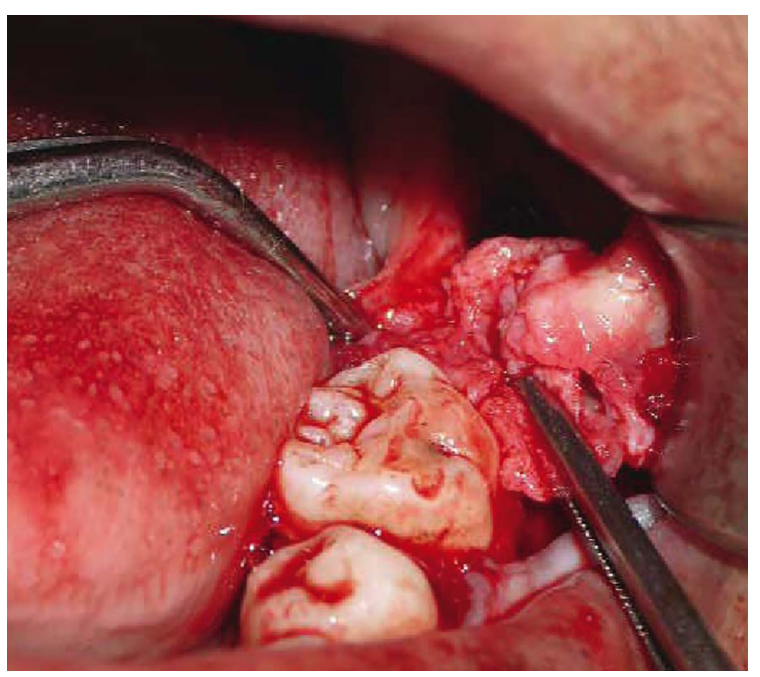

$\triangle$ Fig. 9 : Enucléation de la lésion kystique mandibulaire postérieure gauche.

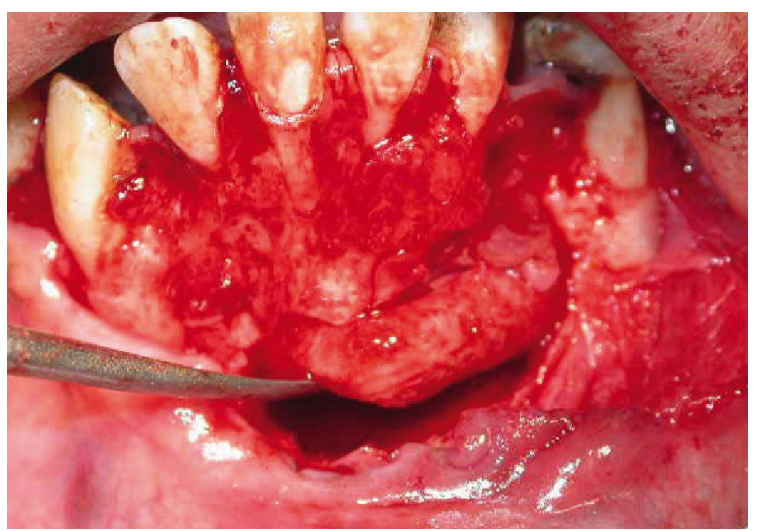

$\triangle$ Fig. 10 : Enucléation chirurgicale de la lésion kystique antérieure.
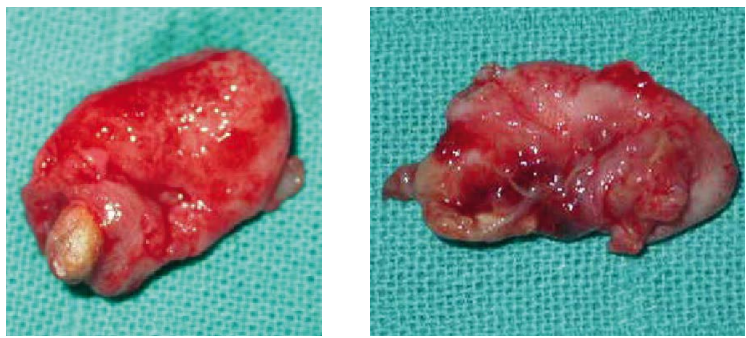

$\triangle$ Fig. 11 : Pièces opératoires. 


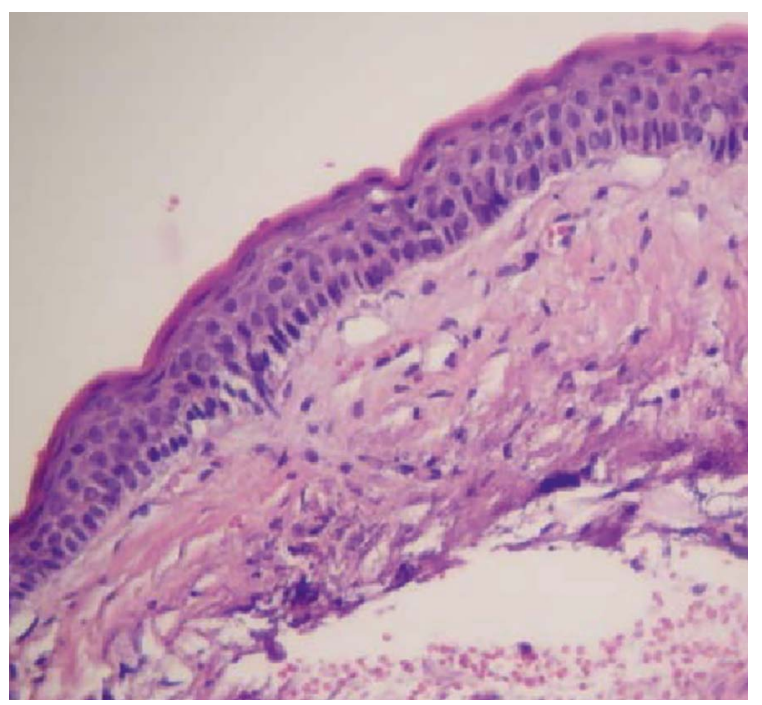

$\triangle$ Fig. 12 : Aspect microscopique de la paroi kystique : cellules parakératosiques en surface, lame basale palissadique et cylindrique.

\section{DISCUSSION}

Le syndrome de GORLIN-GOLTZ est observé de façon égale chez les deux sexes. La majorité des cas a été rapportée dans la race blanche [1].

Les premières manifestations semblent être les kystes maxillaires, pendant la première décennie. Dans un tiers des cas, les signes cutanés débutent à la puberté. Les manifestations cliniques du syndrome de GORLIN-GOLTZ sont très diverses, on note principalement :

\section{Signes maxillo-dentaires}

\section{Les kératokystes odontogéniques}

Approximativement $5 \%$ des kératokystes sont associés à ce syndrome. Ils sont retrouvés chez $80 \%$ des patients avec un syndrome de Gorlin alors qu'ils ne sont présents que chez 5 à $7 \%$ de la population générale $[2,4,5,6,7]$.

\section{Anomalies dentaires}

Les autres anomalies du système stomatologique incluent les malocclusions, les dents incluses, l'ectopie ou l'hétérotopie dentaire et l'agénésie dentaire. Par ailleurs les dents difformes et les dents manquantes (30\% des cas), la susceptibilité à la carie sont plus fréquentes chez les individus atteints du syndrome de Gorlin que chez leurs membres de familles non affectés [8].

\section{Signes cutanéo-muqueux}

Navi et carcinomes basocellulaires

Cliniquement, les naevi se développent souvent en premier et se comportent différemment des carcinomes basocellulaires qui peuvent apparaître et se développer à partir des naevi.
Le développement de carcinome basocellulaire est l'une des caractéristiques les plus problématiques du syndrome de Gorlin, leur nombre varie de quelques uns à plusieurs centaines, et ils ont un large spectre de présentations cliniques qui peut aller d'une claire à sombre papule de consistance dure et de surface plane, à des plaques ulcérées et pigmentées de taille différente $[2,9,10]$.

\section{Puits palmo plantaires}

Les puits ou bien "pits » distinctifs trouvés sur les paumes des mains et les plantes des pieds semblent être pathognomoniques et constituent l'un des critères majeurs de la maladie $[8,11,12]$.

\section{Les kystes épidermoïdes}

Les kystes épidermoïdes larges $(1-2 \mathrm{~cm})$ et souvent multiples ressemblant aux kératokystes odontogènes surviennent sur les membres et le tronc chez environ $50 \%$ des caucasiens atteints du syndrome, chez les Afro-Américains, environ $35 \%$ présentent ces kystes, mais des études supplémentaires sont nécessaires pour évaluer toute différence raciale [2].

\section{Signes osseux}

\section{Calcification de la faux du cerveau}

La calcification de la faux du cerveau est un signe de diagnostic très utile et doit suggérer fortement chez l'enfant la présence du syndrome de Gorlin. Elle peut apparaître très tôt dans la vie, et elle est souvent plus évidente à la fin de l'enfance, et son degré progresse avec l'âge $[2,8]$.

\section{Anomalies costales}

Les anomalies du développement du squelette sont présentes dès la naissance et $70 \%$ des patients atteints de ce syndrome présentent au moins une anomalie squelettique congénitale. La présence de côtes bifides est la manifestation musculo-squelettique la plus caractéristique de la maladie [13, 14].

Ces malformations peuvent donner une forme inhabituelle au thorax, incluant une inclinaison caractéristique vers le bas des épaules. Les côtes bifides sont notées chez environ $6 \%$ de la population générale. Les anomalies costales, avec la cyphoscoliose, provoquent le pectus excavatum (ou thorax en entonnoir ou déformation en creux de la paroi thoracique) $[8,10]$.

\section{Anomalies vertébrales}

Les anomalies des vertèbres cervicales ou thoraciques sont des signes de diagnostic utiles, étant trouvées chez environ $60 \%$ des gens affectés. Les vertèbres C6, C7, TI et $\mathrm{T} 2$ sont les plus fréquemment impliquées $[13,15]$. 


\section{Signes gynécologiques}

\section{Fibromes et fibrosarcomes ovariens}

Initialement, il était difficile de connaître la fréquence absolue des fibromes ovariens associés au syndrome de Gorlin car ils ne se présentent que lorsqu'ils augmentent de volume et deviennent calcifiés et se tordent sur leurs pédicules.

Les fibromes associés au syndrome sont le plus souvent bilatéraux (75\%), multi-nodulaires, calcifiés et les ovaires sont souvent chevauchés médialement. [2, $12,16,17]$.

\section{Hypogonadisme chez l'homme}

5 à $10 \%$ des hommes peuvent présenter des signes d'hypogonadisme hypogonadotrophique tels que l'anosmie, la cryptorchidie, des pilosités pubiennes féminines, la gynécomastie, et/ou des poils peu abondants sur le visage ou le corps. Gorlin en a cité de nombreux exemples. Shanley et al, dans leur enquête, ont noté que $10 \%$ des cas présentaient une anosmie [12].

\section{Signes neurologiques et psychiatriques}

Retard mental

Agénésie du corps calleux,

I Médulloblastome

Méningiomes

\section{DIAGNOSTIC}

$\mathrm{Vu}$ la complexité des signes cliniques retrouvés dans ce syndrome, des critères spécifiques sont nécessaires au diagnostic. Le diagnostic de Neavomatose Baso Cellulaire requiert la présence de 2 critères majeurs ou un critère majeur et 2 mineurs qui sont représentés dans le tableau suivant [18].

\section{Critères diagnostiques de la NBC}

\section{- Critères majeurs :}

- Carcinomes basocellulaires multiples (> 2), ou un carcinome basocellulaire avant l'âge de 20 ans

- Kystes odontogéniques du maxillaire histologiquement prouvés

- Pits palmoplantaires $\geq 3$

- Côtes bifides, fusionnées ou particulièrement évasées

- Atteinte d'un parent du premier degré

\section{- Critères mineurs :}

- Macrocéphalie

- Malformations congénitales orofaciales (une ou plusieurs) : fente labiale ou palatine, bosse frontale, visage grossier, hypertélorisme modéré ou sévère

- Autres anomalies squelettiques : malformation de Sprengel, pectus, syndactylie

- Anomalies radiologiques : selle turcique fermée, anomalies vertébrales : hémi vertèbres, fusion ou allongement des corps vertébraux, défects osseux des mains ou des pieds, petites lacunes osseuses en forme de flamme des mains et des pieds

- Fibrome ovarien

- Médulloblastome

$\triangle$ Les critères diagnostiques de la nævomatose basocellulaire (18).

Eu égard des lésions rapportées dans la littérature, notre patiente présente un critère majeur et 2 critères mineurs, ce qui nous permet d'appuyer le diagnostic du syndrome de Gorlin-Goltz.

\section{PRISE EN CHARGE DES PATIENTS ATTEINTS DE NAEVOMATOSE BASOCELLULAIRE}

\section{L'éducation des patients}

Les patients atteints ont besoin d'informations à-propos du syndrome. Les résultats de plusieurs études épidémiologiques ont montré que les risques de $\mathrm{CBC}$ présentent une forte corrélation avec l'exposition aux rayonnements UV. Ainsi ces patients sont-ils à même d'éviter l'exposition excessive au soleil. Il importe qu'ils portent des lunettes de soleil bloquant les UV à $100 \%$ puisque la peau entourant les yeux (semblable à celle du nez/oreilles) est vulnérable au CBCs.

Les crèmes solaires à indice de protection élevé $($ SPF $30+)$ doivent être appliquées avant de sortir et réappliquée toutes les 2 à 3 heures, et plus fréquemment lors de la natation ou la transpiration [10].

\section{Prise en charge odontologique}

Le rôle du médecin dentiste commence tout d'abord par le diagnostic de la naevomatose basocellulaire. Le tableau de VIGUIER met en évidence l'âge d'apparition des 3 des principaux symptômes de la maladie et montre le rôle primordial du médecin-dentiste dans le dépistage de cette maladie [19]. 


\begin{tabular}{|cccc|}
\hline \multicolumn{4}{c|}{ Évolution de la maladie et étapes de dépistages } \\
\hline & 5 ans & 15 ans \\
Médulloblastome & Kystes épidermoides des maxillaires & Carcinomes basocellulaires \\
Pédiatre & Chirurgien-dentiste & Dermatologue \\
Pas de signe & Dépistage & Diagnostic établi \\
\hline
\end{tabular}

$\triangle$ Tableau de Viguier mettant en évidence l'âge d'apparition de 3 des principaux symptômes de la maladie et montre le rôle primordial du chirurgien-dentiste dans le dépistage du syndrome de Gorlin-Goltz.

\section{TRAITEMENT DES KÉRATOKYSTES}

Le traitement des kératokystes odontogéniques est chirurgical. Les objectifs du traitement chirurgical des kératokystes est l'éradication de la totalité de la lésion ainsi que la réduction du potentiel de récurrence. Diverses techniques chirurgicales ont été proposées pour traiter les kératokystes. Celles-ci vont des procédures plus conservatrices (par exemple, énucléation, marsupialisation), à des approches plus agressives (par exemple la résection en bloc) [20].

Le choix thérapeutique se fait en fonction de la topographie des kératokystes, de leur extension, de leur aspect évolutif, primitif ou récidivant. [21, 22, 23, 24, 25].

Le traitement chirurgical des kératokystes du cas clinique a consisté en une simple énucléation suivie d'un curetage minutieux.

\section{PRONOSTIC}

Les kératokystes odongéniques occupent une place à part parmi les kystes odontogènes. On les considère actuellement comme des tumeurs bénignes du fait de leur activité mitotique plus élevée et de l'importance de la lyse osseuse engendrée. Ils présentent un risque accru de transformation maligne en carcinome épidermoïde.

Ces lésions présentant un potentiel de récidive important même à distance du traitement primaire (jusqu'à 10 ans plus tard), il est recommandé de procéder à des examens de contrôle annuels. Les taux de récidive les plus faibles sont observés après les interventions radicales, les plus élevés sont enregistrés après de simples énucléations [1].

\section{CONCLUSION}

Le syndrome de Gorlin et Goltz est une affection rare mais pas exceptionnelle qui doit être connu par le médecin-dentiste. Il est défini classiquement par la triade composée de naevi baso-cellulaires, de kératokystes des maxillaires et de malformations squelettiques. La prise en charge thérapeutique reste simplement symptomatique.

Le risque carcinologique de ce syndrome en fait sa gravité, imposant un dépistage précoce et une surveillance régulière et prolongée des patients et de leurs descendances.

Conflits d'intérêt : aucun. 


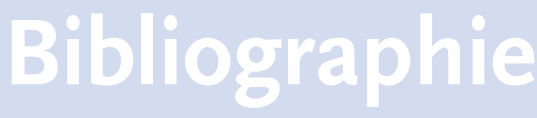

[1] Manfredi M, et al. Nevoid basal cell carcinoma syndrome : a review of the literature. Int. J. Oral. Maxillofac. Surg. 2004; 33: 117-124.

[2] Gorlin RJ. Nevoid Basal Cell Carcinoma (Gorlin) Syndrome. In : Ruggieri M, Pascual-Castroviejo I, Di Rocco C, editors. Neurocutaneous Disorders : Phakomatoses and Hamartoneoplastic Syndromes. New York: Springer-Verlag ; 2008 ; pp. 669-694.

[3] Lomuzziol. Nevoid baso cell carcinoma syndrome (Gorlinsyndrome). Orphanet journal of rare diseases, 2008 Nov25, 3, 32.

[4] Bornsteinm M, et al. Le kératokyste odontogène : kyste odontogène ou tumeur bénigne ? Rev. Mens. Suisse Odonto-Stomato., 2005, 115, 2, 123-128.

[5] Ruhin-Poncet B, et al. Kératokystes (ou tumeurs odontogéniques kératokystiques). Rev. Stomatol. Chir. Maxillo-fac. 2011 Apr; $112(2): 87-92$.

[6] Rivetj, et al. Syndrome de Gorlin-Goltz. À propos d'un kyste du maxillaire. Rev. Stomatol. Chir. Maxillo-fac., Oct 2000, 101, 4, 194-196.

[7] Haitami S, et al. Kératokystes odontogènes multiples non syndromiques : une nouvelle observation. Med Buccale Chir Buccale 2013 ; $19: 259-262$.

[8] Farndon P. Gorlin syndrome : Nevoid basal cell carcinoma syndrome. In : Cassidy
SB, Allanson JE editors. Management of genetic syndromes, third Edition. Wiley-Blackwell ; 2010 ; pp. 413-428.

[9] Crutchfieldc E, et al. What Syndrome Is This? Pedriatr. Dermatol., 2000, 17, 6, 484-6.

[10] Lomuzziol. Nevoid basal cell carcinoma syndrome (Gorlinsyndrome). Orphanet journal of rare diseases, 2008 Nov 25, 3, 32.

[11] Didier B. Manifestations dermatologiques des maladies du système hématopoïétique et oncologie dermatologique. Paris : SpringerVerlag, 2009. Derm. med. vol. 3, 250 p.

[12] Gorlin RJ, et al. Multiple nevoid basal cell carcinoma syndrome. In : Syndromes of the Head and Neck (2001), 4th ed. Oxford : Oxford University Press, pp. 444-453.

[13] Kimonisv E, et al. Clinical manifestations in 105 persons with nevoid basal cell carcinoma syndrome. Am. J. Med. Genet., 1997, 69, 229-308.

[14] Rupprechtm, et al. Skeletal and dermatological manifestations of the nevoid Basal cell carcinoma syndrome (Gorlin-Goltz syndrome). Results of 8 patients in 12 years Röfö., 2007 Jun, 179, 6, 618-26.

[15] Kimonis VE, et al. Radiological features in 82 patients with nevoid basal cell carcinoma (NBCCS or Gorlin) syndrome. Genet Med. 2004 Nov-Dec ; 6 (6) : 495-502.

[16] Evansd G, et al. Complications of the nevoid basal cell carcinoma syndrome : result of a population based study. J. Med. Genet., 1993, 30, 460-4.
[17] Fonsecar B, et al. Bilateral ovarian fibromasin nevoid basal cell carcinoma syndrome. Radiology, 2008,246,318-21.

[18] Didier B. Manifestations dermatologiques des maladies du système hématopoiétique et oncologie dermatologique. Paris : SpringerVerlag, 2009. Derm. med. vol. 3, 250 p.

[19] Viguier PA, et al. Le diagnostic et la prise en charge dentaire du syndrome de Gorlin. J. Soc. Odontol. Paris, jun 2007, 6, 14-15.

[20] Williamst P, et al. Surgical management of the odontogenic keratocyst : aggressive approach. J Oral Maxillofac. Surg., sep 1994, 52 , 9, 964-6.

[21] Tolstunov L, et al. Surgical Treatment Algorithm For Odontogenic Keratocyst. J Oral Maxillofac Surg. 2008 ; 66 : 1025-1036.

[22] Letouxg, et al. Approche chirurgicale des kératokystes odontogènes : à propos de 2 cas cliniques. Med. buccalechir. buccale, jan 2001, 7, 1, 33-41.

[23] Madrasj, et al. Keratocystic odontogenic tumour : reclassification of the odontogenic keratocyst from cyst to tumour. J Can Dent Assoc., 2008Mar, 74,2,165.

[24] Perrinj $P$, et al. Très grands kératokystes mandibulaires : mise au point. Rev. Stomatol. Chir. Maxillo-fac., Sep 2002, 103, 4, 207-220.

[25] Tonietto L, et al. Enucleation and liquid nitrogen cryotherapy in the treatment of keratocystic odontogenic tumors: a case series. J Oral Maxillofac Surg. 2011 Jun ; 69 (6) : e112-7. 